\title{
A Survey on Radio Resource Allocation for V2X Communication
}

\author{
Ahlem Masmoudi (D), Kais Mnif, and Faouzi Zarai \\ NTS'COM Research Unit, National School of Electronics and Telecommunications, University of Sfax, Sfax, Tunisia \\ Correspondence should be addressed to Ahlem Masmoudi; ahlem.massmoudi@gmail.com
}

Received 1 May 2019; Revised 22 September 2019; Accepted 27 September 2019; Published 24 October 2019

Guest Editor: Donghyun Kim

Copyright ( $) 2019$ Ahlem Masmoudi et al. This is an open access article distributed under the Creative Commons Attribution License, which permits unrestricted use, distribution, and reproduction in any medium, provided the original work is properly cited.

\begin{abstract}
Thanks to the deployment of new techniques to support high data rate, high reliability, and QoS provision, Long-Term Evolution (LTE) can be applied for diverse applications. Vehicle-to-everything (V2X) is one of the evolving applications for LTE technology to improve traffic safety, to minimize congestion, and to ensure comfortable driving which requires stringent reliability and latency requirements. As mentioned in the 3rd Generation Partnership Project (3GPP), LTE-based Device-to-Device (D2D) communication is an enabler for V2X services to meet these requirements. Therefore, radio resource management (RRM) is important to efficiently allocate resources to V2X communications. In this paper, we present the V2X communications, their requirements and services, the V2X-based LTE-D2D communication modes, and the existing resource allocation algorithms for V2X communications. Moreover, we classify the existing resource allocation algorithms proposed in the literature and we compare them according to selected criteria.
\end{abstract}

\section{Introduction}

Internet of Things (IoT) include billions of intelligent objects which are regarded as a part of the future Internet $[1,2]$. IoT is a growing number of things (i.e., physical objects) that are wirelessly connected to the Internet via smart sensors. IoT enables these things to interact and exchange data in an efficient way without human intervention. The 3rd Generation Partnership Project (3GPP) Long-Term Evolution (LTE) is one of the main strengths of the IoT which seeks to cover all the IoT applications. Vehicle-to-Everything (V2X) communication is an important main area of IoT. Recently, 3GPP has made Release 14 for LTE-based V2X communication (cellular V2X) in order to improve the safety, the efficiency, and the comfort of intelligent transportation systems (ITS). The term V2X refers to the vehicle to vehicle (V2V), vehicle to infrastructure (V2I), vehicle to pedestrian (V2P), and vehicle to Network (V2N) communications [3] which require a high reliability and low latency.

On the other hand, in Release 16, 3GPP is working near the growth of New Radio (NR) V2X, constructing over 5G NR that was standardized in Release 15 of 3GPP. NR V2X should support advanced V2X services that necessitate much more stringent QoS assurances compared to services that may be supported by C-V2X $[4,5]$. For both evolutionary, the $5 \mathrm{G}$ NR and C-V2X are designed to progress lower endto-end latency and reliability services and to support services that require high throughput. But, their design methodologies meaningfully vary. 3GPP does not carry out a similar constraint on NR V2X. V-UEs equipped with NR V2X can interconnect with C-V2X devices. Nevertheless, this will be reached through a dual-radio access system-one radio for NR V2X and another for C-V2X [6].

The proximity service (ProSe) known as a Device-toDevice (D2D) communication, defined in Release 12, refers to a direct communication between two or more devices in proximity to each other rather than travelling through the eNodeB [7]. Therefore, several kinds of advantages can be offered due to the proximity, reuse, and hop gain. D2D communications were initially proposed to improve network performance (i.e., enhancing spectrum utilization, improving UEs throughput, increasing cellular capacity, and extending UEs battery lifetime) in cellular technologies [8].

Since V2X technologies have stringent reliability and latency requirements [9], it was declared in Release 14 that the D2D communication can be applied in vehicular 
technologies to support $\mathrm{V} 2 \mathrm{~V}$ communications. As $\mathrm{V} 2 \mathrm{X}$ is based on the D2D communication, resources are allocated in the V-UEs either in the overlay mode or in the underlay mode. For that reason, radio resource management (RRM) plays an important role in V2X system performances.

In this paper, we provide a wide review of available literature for resource allocation algorithms in V2X services based on D2D communication. Moreover, we provide a comprehensive comparison and classification of V2X resource allocation algorithms in terms of numerous aspects. The paper is structured as follows: The V2X services are described in Section 2. The V2X communication modes and the LTE-V-based D2D communication are introduced, respectively, in Section 3 and Section 4. A review and classification of the existing resource allocation algorithms for V2X communication are addressed in Section 5. We discuss and conclude this paper in Section 6 and Section 7.

\section{V2X Requirements and Services}

3GPP defines 27 use cases for V2X services in Release 14 that can be divided into safety and nonsafety V2X services [10]. Therefore, the transmission of V2X messages should be classified according to the message type (e.g., safety vs. nonsafety). The safety services-related use cases aim to avoid automobile accidents and to protect the property and life, which require high reliability and short delay (e.g., vehicle platooning and automated driving). The nonsafety use cases aim to improve the driving experience to be more comfortable and efficient (e.g., mobile high data rate entertainment and traffic management). Therefore, the non-safety and the safety V2X services have different latency and packet size requirements [11].

Five categories of service requirements of this use cases are defined as follows $[9,10]$ :

(i) Speed: the maximum absolute velocity $160 \mathrm{~km} / \mathrm{h}$ and the maximum relative velocity $280 \mathrm{~km} / \mathrm{h}$ shall be supported. The maximum relative speed of $500 \mathrm{~km} / \mathrm{h}$ is supported for the possible scenario without speed limitation.

(ii) Communication range: the effective distance is larger than the distance calculated as the ample response time (e.g., 4 seconds) for the drivers to avoid collision according maximum relative speed.

(iii) Latency/reliability: the maximum end-to-end latency between two UEs supporting V2V/V2P/V2I applications shall be $100 \mathrm{~ms}$. The minimum radio layer reliability should be supported without retransmissions of application-layer messages in the effective distance and the restricted latency.

(iv) Message size: the variable length of a periodically message between two UEs supporting V2X applications is about $50-300$ bytes. The message size of event-triggered messages can be up to 1200 bytes.

(v) Message generation period: the minimum message generation period can be $100 \mathrm{~ms}$.
2.1. Safety-Critical V2X Services. The safety-critical V2X services aim to reduce the potential accident and the risk to pedestrians and to deteriorate the possibility of life dead for V-UEs. The information shared between pedestrians, Road Side Unit (RSU), and vehicles (i.e., vehicle, speed, position, and distance) is usually short broadcast messages which impose stringent requirements on latency, packet loss, and reliability. Two types of safety V2X transmissions messages have been standardized, the periodic transmission and event-triggered transmission. The periodic transmissions among V2V-UEs (e.g., the cooperative awareness messages (CAMs) are short broadcast messages periodically transmitting aware information of position, presence, speed, and directions among vehicles and their neighbors. The event-triggered transmission among V2X-UEs (i.e., decentralized environmental notification messages (DENMs)) is short broadcast messages to alert road users about road status. Both periodic transmission and event-triggered transmission can be operated over the LTE-Uu and the PC5 interfaces [12].

2.2. Nonsafety V2X Services. The nonsafety V2X services essentially focus on management application, control congestion, traffic efficiency, and entertainment; these services enable more comfortable and efficient driving experience. Nonsafety V2X applications aim to improve traffic coordination, assistance, and vehicle traffic flow. In addition, they also provide entertainment video, maps, and updated local information. Therefore, a large amount of V-UEs sensed data sent to neighbors or to infrastructure. These services have no strict requirements on reliability and latency.

\subsection{V2X Requirements}

2.3.1. Reliability Requirement. The reliability requirement defined in different works is generally interpreted from either the outage probability (OP) or the packet reception ratio (PRR).

The PRR is the ratio of successful reception among the total number of the vehicle transmitter neighbors, which are calculated as follows:

$$
\begin{aligned}
\operatorname{PRR}_{u}(t) & =\frac{1}{N_{u}(t)} \sum_{j=1}^{N_{u}(t)} 1\left\{\gamma_{j}^{(u)}(t) \geq \gamma_{\mathrm{th}}\right\}, \\
\gamma_{j}^{(u)}(t) & =\sum_{k=1}^{N_{R B}} \frac{s_{k}^{u}(t) P_{u}(t)\left|H_{u j}^{k}(t)\right|^{2}}{\sigma^{2}+\sum_{m=1}^{N_{L}} s_{m}^{u}(t) P_{j}(t)\left|H_{m}^{k}(t)\right|^{2}},
\end{aligned}
$$

where $N_{\mu}(t)$ denotes the number of the vehicles neighbors of the $u^{\text {th }}$ vehicle at time $t, \gamma_{j}^{(u)}(t)$ is the receiving signalto-interference-plus-noise ratio (SINR) of the $j^{\text {th }}$ vehicle among $N_{u}(t), \gamma_{\text {th }}$ is the SINR threshold, $P_{u}(t)$ is the transmit power of $u^{\text {th }}$ vehicle, $\sigma^{2}$ is the power of additive white Gaussian noise, $N_{L}$ is the number of interfering 
vehicles, $s_{k}^{u}(t)$ is the RBs allocation at time $t$, and $s_{k}^{u}(t)= \begin{cases}1, & \text { if } k^{\text {th }} R B \text { is allocated to user } u \text { at time } t, \\ 0, & \text { otherwise. }\end{cases}$

The OP is the most utilized which is the probability that $N_{u}$ error-free bits cannot be sent by any coding pattern which is computed as follows:

$$
p_{u}^{\text {out }} \triangleq \operatorname{Pr}\left\{\sum_{i=1}^{E_{u}^{\text {all }}} \rho \log \left(1+\gamma_{i}\right)<N_{u}\right\}
$$

where $E_{u}^{\text {all }}$ is the RBs number that are allocated to the $u^{\text {th }}$ $\mathrm{V}$-UE, $\rho$ is the number of complex symbols in the RB, and $\gamma_{i}$ is the SINR of the $i^{\text {th }} \mathrm{RB}$.

2.3.2. Latency Requirement. The latency requirement in some studies is interpreted by the latency constraint that allows $\mathrm{V}$-UEs to allocate their required $\mathrm{RBs}$ before the maximum tolerable latency according to scheduling time unit number. Most of the studies designate that C-V2X can support safety services reliably that required an end-to-end latency of nearby 100 milliseconds (msec).

\section{V2X Communication Modes}

To support both safety/nonsafety V2X applications, the 3GPP standard provides two radio interfaces for LTE-Vehicle (LTE-V) $[13,14]$ : the Uu cellular interface that supports V2I communications (e.g., enhanced Multimedia Broadcast Multicast Service (eMBMS) and the PC5 interface (direct LTE sidelink) that supports V2V communications as shown in Figure 1.

\subsection{LTE-Cellular Communication Mode Using Uu Interface.} LTE-Cellular communication refers to the communication mode between V-UEs and the eNB, which is suitable for nondelay tolerant use cases (e.g., mobility services and situational awareness) using the LTE technologies. The eNB receives unicast messages from $\mathrm{V}$-UEs and rebroadcasts them for all $\mathrm{V}$-UEs receivers in the pertinent area using eMBMS. Based on coordination between multiple cells, MBMS aims to reduce the latency and to improve the performance of the cell edge.

3.2. LTE-D2D Communication Mode Using PC5 Interface. LTE-D2D communication refers to the communication mode between two V-UEs in proximity to each other, bypassing the eNB. This mode is appropriate for V2V safety services that require low latency delay (e.g., advanced driverassistance systems (ADAS). This communication mode is based on Release 12 proximity services (ProSe) which exploit direct communication between neighboring devices. Two transmission modes are introduced in Release 12 (mode 1 and mode 2) for LTE sidelink (or D2D communication) public safety. These modes are designed in order to prolong the devices' battery lifetime at the cost of increasing the latency. Therefore, mode 1 and mode 2 are not convenient for V2X applications since connected vehicles require low

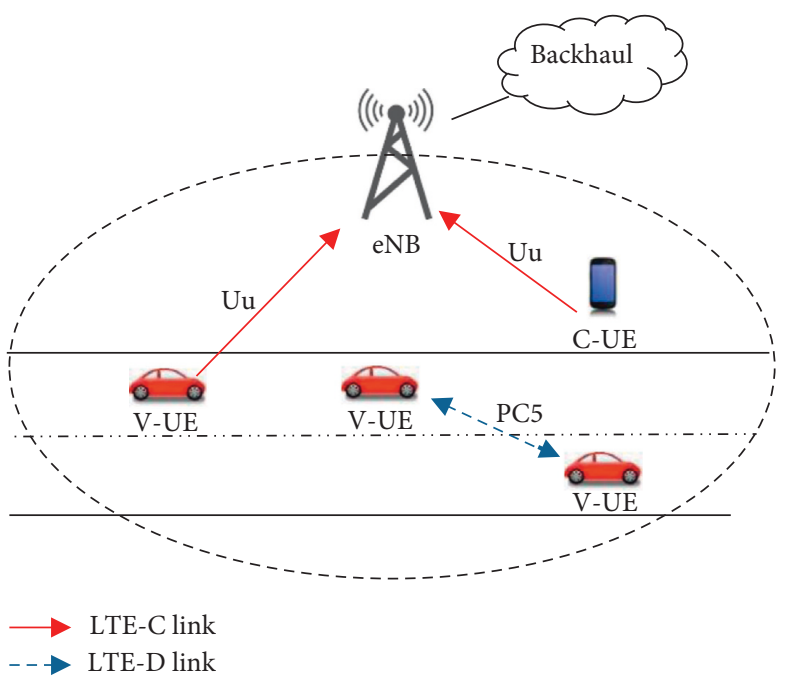

FIGURE 1: Radio interfaces for V2X communications.

latent and highly reliable V2X communications. Recently, two new sidelink communication modes are introduced in Release 14, which are typically intended for V2V communications, the mode 3 and the mode 4 . In mode 3 , the radio resources used by the direct $\mathrm{V} 2 \mathrm{~V}$ communications and the interference management are managed and assisted by the cellular infrastructure (e.g., eNB). However, vehicles autonomously select and assign the radio resources in mode 4 for their direct V2V communications without infrastructure assistance (i.e., this mode can be operated out of cellular coverage).

In this paper, we investigate the radio resource allocation in mode 3 for the V2X services based on D2D communication in LTE cellular network.

\section{LTE-V-Based D2D Communication}

Since there is a similarity between the localized nature of $\mathrm{V} 2 \mathrm{~V}$ and D2D communications, it is mentioned in Release 14 that the D2D communication can be useful in vehicular networks. The V2V/D2D communication can allocate the cellular resources in orthogonal or nonorthogonal way.

4.1. V2V-Based D2D Communication Modes. There are two $\mathrm{V} 2 \mathrm{~V}$ communication modes: the overlay mode (which is the orthogonal resource allocation) and the underlay mode (which is the nonorthogonal one) as shown in Figure 2.

In the overlay mode, specific radio resources from cellular resources are dedicated to the D2D/V2V communication. Thus, the C-UEs cannot achieve the full capacity of the eNB; consequently, this mode decreases the spectrum utilization. The advantage of the overlay mode is that the interference between C-UEs and V2V-UEs does not need to be managed.

In the underlay mode, the eNB allows V2V-UEs and $\mathrm{C}$-UEs to share the same radio resources, which can achieve a greatest spectrum efficiency. However, the eNB needs to manage the strong interference among V2V-UEs communications and the C-UEs communications. 


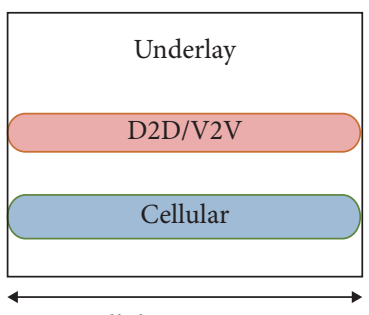

Cellular spectrum

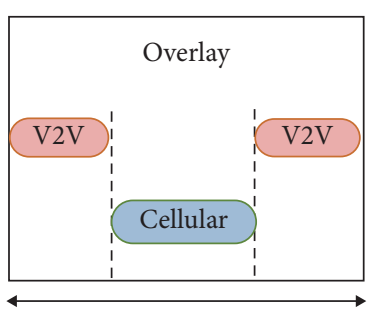

Cellular spectrum
FIgURE 2: Underlay vs overlay modes.

Both of the overlay and the underlay modes can utilize either the Downlink (DL) or the Uplink (UL) subframe. The set of resources that will be allocated to $\mathrm{V} 2 \mathrm{~V}$ communication are chosen from the UL subframe owing to their minor peak-to-average power ratio (PAPR) and because it is less utilized than the DL subframe.

4.2. Nonorthogonality among V-UEs. Orthogonal multiple access (OMA) techniques are established by all of the current cellular mobile networks (i.e., LTE, LTE-A) such as orthogonal frequency division multiple access (OFDMA) and time-division multiple access (TDMA) where a single user can be served in each orthogonal resource block (RB) in OFDMA subcarriers. Nevertheless, these techniques cannot meet the great demands of the future radio access networks [15]. Therefore, the nonorthogonal multiple access (NOMA) is suggested as a candidate for $5 \mathrm{G}$ cellular mobile systems which becomes a significant principle for the $5 \mathrm{G}$ radio access techniques [16]. NOMA can be deployed in the future and the existing mobile systems due to its compatibility with other technologies. Unlike OMA and without needing any modifications to the LTE RBs, NOMA can serve multiple users on the same RB (in the same frequency and time domain) as shown in Figure 3. Therefore, the NOMA system throughput can be expressively greater than that of the OMA. The NOMA technique is also applied to cellular vehicular network in order to achieve low latency and high reliability and to reduce resource collision [17].

\section{RRM for V2X Services over LTE}

RRM in LTE includes a diversity of techniques and procedures, such as radio resource allocation and packet scheduling. Nowadays, resource allocation in LTE-V is one of the most discussed topics. The V-UEs can communicate using either direct link or cellular link. They can also operate either in the underlay or in the overlay modes. The majority of existing related works suggest using the cellular spectrum for both V-UEs and C-UEs. Since in the overlay mode, a set of RBs are dedicated to V-UEs using the direct link, the CUEs cannot achieve the full spectrum capacity. Therefore, studies based on the overlay mode aim to avoid the waste of resources. On the other hand, C-UEs and V-UEs share the same RBs in the underlay mode, which reach a best spectrum efficiency but the huge interference could occur among C-UEs and V-UEs. Several V2X resource allocation algorithms have been investigated in the literature. The majority

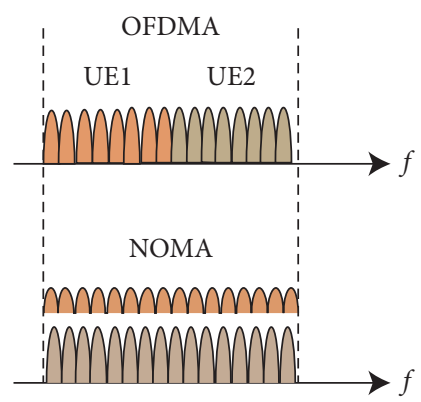

Figure 3: Difference between OMA and NOMA.

of these algorithms underlaying C-UEs need to deal interferences among C-UEs and V-UEs. In this section, we demonstrate an overview classification on V2X resource allocation algorithm existing in the literature as shown in Figure 4.

Then, we provide a comprehensive classification and comparison of the V2X resource allocation algorithms existing in the literature in terms of numerous aspects (e.g., communication mode and allocation process). Moreover, we study the existing effort in LTE-V2X resource allocation algorithms in-band communication over the underlay mode and the overlay mode where the allocation of RBs is done by the eNB (mode 3).

5.1. Underlaying Resource Allocation in V2X Services. Early-proposed V2X radio resource allocation algorithms in LTE- $V$ environments suggest reusing cellular spectrum for V2X communications. Nowadays, the majority of existing resource allocation work is dedicated to the underlaying V2X communications in cellular networks. These works typically study the interference problems between V-UEs and C-UEs due to RBs shared between them. The existing resources allocation algorithms designed in the underlay mode can be categorized according to several criteria. We classify these algorithms according to RBs sharing process, the RBs sharing based on user pairing, user clustering/grouping, and user geographic location.

5.1.1. RBs Sharing-Based User Pairing. Most of the underlaying resource allocation algorithms allow UEs to share the same RBs based on user pairing where the eNB finds the optimal combination between at least two UEs to share the same RB. The main objective is to allow at least one V-UE using direct link to share the same RB with only one C-UE or with only one V-UE using cellular link. The comparison of these algorithms is summarized in Table 1.

In [18], Zhang et al. proposed a novel resource sharing of the underlaying communication mode for vehicular networks which aims to increase throughput of the vehicular network through efficient interference management protocols. Different V2I and V2V communication links are allowed to access the same resources for their data transmission. The resource-sharing problem was formulated as a resource allocation optimization problem, 


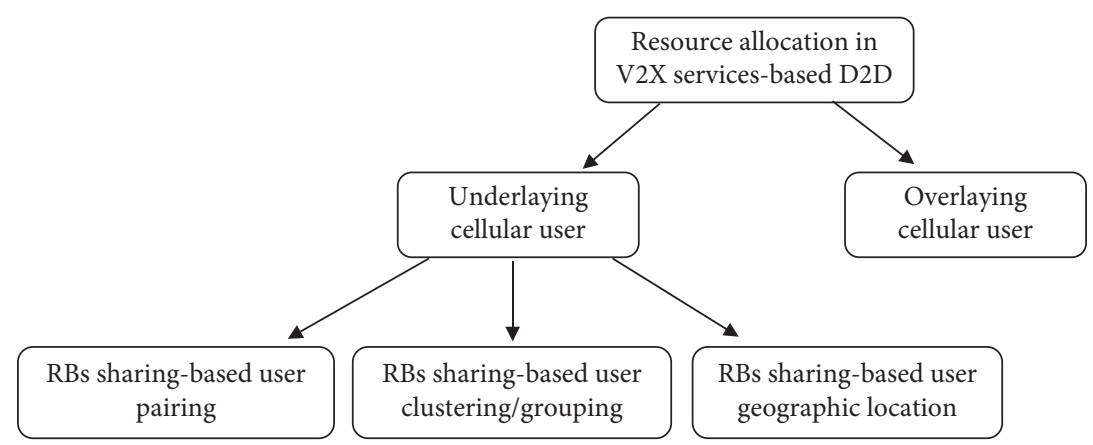

FIGURE 4: Resources allocation in V2X services classification.

TABLE 1: Comparison of the existing underlaying RBs sharing-based user pairing V2X services.

\begin{tabular}{|c|c|c|c|c|c|c|c|c|c|}
\hline Ref. & $\begin{array}{l}\text { Unicast/ } \\
\text { broadcast }\end{array}$ & Objectives & Scenarios & User types & $\begin{array}{l}\text { Allocation } \\
\text { constraints }\end{array}$ & $\begin{array}{c}\text { Power } \\
\text { control/ } \\
\text { allocation } \\
\end{array}$ & $\begin{array}{l}\text { Allocation } \\
\text { process }\end{array}$ & Methods/theory & $\begin{array}{c}\mathrm{RB} \\
\text { sharing }\end{array}$ \\
\hline$[18]$ & Broadcast & $\begin{array}{l}\text { Increase } \\
\text { throughput }\end{array}$ & Road & $\begin{array}{l}\text { V2V-UEs } \\
\text { V2I-UEs }\end{array}$ & $\mathrm{PF}$ & - & $\begin{array}{l}\text { Orthogonal RBs } \\
\text { are allocated to } \\
\text { each user type }\end{array}$ & Graph theory & $\begin{array}{l}1 \mathrm{~V} 2 \mathrm{~V}-\mathrm{UE} \\
1 \mathrm{~V} 2 \mathrm{I}-\mathrm{UE}\end{array}$ \\
\hline [19] & Unicast & $\begin{array}{l}\text { Maximize sum } \\
\text { rate fairness; } \\
\text { minimize } \\
\text { latency } \\
\text { reliability }\end{array}$ & Urban & $\begin{array}{l}\text { C-UEs } \\
\text { V-UEs }\end{array}$ & $\begin{array}{c}\text { OP } \\
\text { SINR } \\
\text { Latency }\end{array}$ & PC & $\begin{array}{l}\text { Orthogonal RBs } \\
\text { are allocated to } \\
\text { each user type }\end{array}$ & $\begin{array}{l}\text { An interior point } \\
\text { method } \\
\text { Hungarian algorithm }\end{array}$ & $\begin{array}{l}1 \mathrm{C}-\mathrm{UE} \\
1 \mathrm{~V}-\mathrm{UE}\end{array}$ \\
\hline$[20]$ & Unicast & $\begin{array}{l}\text { Maximize sum } \\
\text { rate fairness; } \\
\text { minimize } \\
\text { latency } \\
\text { reliability }\end{array}$ & Urban & $\begin{array}{l}\text { C-UEs } \\
\text { V-UEs }\end{array}$ & $\begin{array}{c}\text { OP } \\
\text { SINR } \\
\text { Latency }\end{array}$ & $\mathrm{PA}$ & $\begin{array}{l}\text { Orthogonal RBs } \\
\text { are allocated to } \\
\text { each user type }\end{array}$ & $\begin{array}{c}\text { Karush-Kuhn-Tucker } \\
\text { theory } \\
\text { Dual decomposition } \\
\text { method } \\
\text { Hungarian algorithm }\end{array}$ & $\begin{array}{l}1 \mathrm{C}-\mathrm{UE} \\
1 \mathrm{~V}-\mathrm{UE}\end{array}$ \\
\hline [21] & Unicast & $\begin{array}{l}\text { Maximize sum } \\
\text { rate fairness; } \\
\text { minimize } \\
\text { latency } \\
\text { reliability }\end{array}$ & Urban & $\begin{array}{l}\text { C-UEs } \\
\text { V-UEs }\end{array}$ & $\begin{array}{c}\text { OP } \\
\text { SINR } \\
\text { Latency }\end{array}$ & PA & $\begin{array}{l}\text { Orthogonal RBs } \\
\text { are allocated to } \\
\text { C-UEs; } \\
\text { nonorthogonal } \\
\text { RBs are } \\
\text { allocated to V- } \\
\text { UEs }\end{array}$ & $\begin{array}{l}\text { Perron-Frobenius } \\
\text { theory } \\
\text { Interior point method }\end{array}$ & $\begin{array}{l}1 \mathrm{C}-\mathrm{UE} \\
\mathrm{N} \text { V-UEs }\end{array}$ \\
\hline$[22]$ & Unicast & $\begin{array}{l}\text { Maximize sum } \\
\text { rate fairness; } \\
\text { minimize } \\
\text { latency } \\
\text { reliability }\end{array}$ & Urban & $\begin{array}{l}\text { C-UEs } \\
\text { V-UEs }\end{array}$ & $\begin{array}{c}\text { OP } \\
\text { SINR } \\
\text { Latency }\end{array}$ & $\mathrm{PA}$ & $\begin{array}{l}\text { Orthogonal RBs } \\
\text { are allocated to } \\
\text { C-UEs; } \\
\text { nonorthogonal } \\
\text { RBs are } \\
\text { allocated to V- } \\
\text { UEs }\end{array}$ & $\begin{array}{l}\text { Matching theory } \\
\text { Interior point method }\end{array}$ & $\begin{array}{l}1 \text { C-UE } \\
\text { N V-UEs }\end{array}$ \\
\hline [23] & Broadcast & $\begin{array}{l}\text { Maximize the } \\
\text { number of } \\
\text { concurrent } \\
\text { V2V } \\
\text { transmissions }\end{array}$ & $\begin{array}{l}\text { Urban } \\
\text { freeway }\end{array}$ & V-UEs & SINR & - & $\begin{array}{l}\text { Nonorthogonal } \\
\text { RBs are } \\
\text { allocated to V- } \\
\text { UEs }\end{array}$ & $\begin{array}{l}\text { Perron-Frobenius } \\
\text { theory }\end{array}$ & N V-UEs \\
\hline$[24]$ & Unicast & $\begin{array}{l}\text { Maximize } \\
\text { throughput; } \\
\text { minimize } \\
\text { latency }\end{array}$ & Urban & $\begin{array}{l}\text { C-UEs } \\
\text { Safety and } \\
\text { nonsafety } \\
\text { V-UEs }\end{array}$ & $\begin{array}{l}\text { SINR } \\
\text { Latency }\end{array}$ & - & $\begin{array}{l}\text { Orthogonal RBs } \\
\text { are allocated to } \\
\text { each user type }\end{array}$ & $\begin{array}{l}\text { Hypergraph matching } \\
\text { theory }\end{array}$ & $\begin{array}{c}1 \text { C-UE } \\
1 \text { safety } \\
\text { V-UE } \\
1 \\
\text { nonsafety } \\
\text { V-UE }\end{array}$ \\
\hline [25] & Unicast & $\begin{array}{l}\text { Maximize } \\
\text { throughput } \\
\text { reliability }\end{array}$ & $\begin{array}{l}\text { Multilane } \\
\text { freeway }\end{array}$ & $\begin{array}{l}\text { V2V-UEs } \\
\text { V2I-UEs }\end{array}$ & $\mathrm{OP}$ & PA & $\begin{array}{l}\text { Orthogonal RBs } \\
\text { are allocated to } \\
\text { each user type }\end{array}$ & Hungarian method & $\begin{array}{l}1 \mathrm{~V} 2 \mathrm{~V}-\mathrm{UE} \\
1 \mathrm{~V} 2 \mathrm{I}-\mathrm{UE}\end{array}$ \\
\hline
\end{tabular}


TABle 1: Continued.

\begin{tabular}{|c|c|c|c|c|c|c|c|c|c|}
\hline Ref. & $\begin{array}{l}\text { Unicast/ } \\
\text { broadcast }\end{array}$ & Objectives & Scenarios & User types & $\begin{array}{l}\text { Allocation } \\
\text { constraints }\end{array}$ & $\begin{array}{c}\text { Power } \\
\text { control/ } \\
\text { allocation }\end{array}$ & $\begin{array}{c}\text { Allocation } \\
\text { process }\end{array}$ & Methods/theory & $\begin{array}{c}\mathrm{RB} \\
\text { sharing }\end{array}$ \\
\hline [26] & Unicast & $\begin{array}{l}\text { Reliability; } \\
\text { maximize the } \\
\text { ergodic } \\
\text { capacity }\end{array}$ & $\begin{array}{l}\text { Multi- } \\
\text { lane } \\
\text { freeway }\end{array}$ & $\begin{array}{l}\text { V2V-UEs } \\
\text { V2I-UEs }\end{array}$ & $\mathrm{OP}$ & PA & $\begin{array}{l}\text { Orthogonal RBs } \\
\text { are allocated to } \\
\text { each user type }\end{array}$ & Hungarian method & $\begin{array}{l}1 \mathrm{~V} 2 \mathrm{~V}-\mathrm{UE} \\
1 \mathrm{~V} 2 \mathrm{I}-\mathrm{UE}\end{array}$ \\
\hline$[27]$ & Broadcast & $\begin{array}{l}\text { Maximize the } \\
\text { C-UEs } \\
\text { information } \\
\text { rate; guarantee } \\
\text { reliability/ } \\
\text { latency } \\
\text { requirement of } \\
\text { V-UEs }\end{array}$ & Urban & $\begin{array}{l}\text { C-UEs } \\
\text { V-UEs }\end{array}$ & $\begin{array}{l}\text { Latency } \\
\text { OP } \\
\text { SINR }\end{array}$ & PA & $\begin{array}{l}\text { Orthogonal RBs } \\
\text { are allocated to } \\
\text { each user type }\end{array}$ & $\begin{array}{c}\text { Lagrange dual } \\
\text { decomposition method } \\
\text { Binary search method } \\
\text { Subgradient iteration } \\
\text { method }\end{array}$ & $\begin{array}{l}1 \mathrm{C}-\mathrm{UE} \\
1 \mathrm{~V}-\mathrm{UE}\end{array}$ \\
\hline [28] & Unicast & $\begin{array}{c}\text { Maximize } \\
\text { throughput (C- } \\
\text { UEs/nonsafety } \\
\text { V-UEs); } \\
\text { guarantee QoS } \\
\text { demand (W- } \\
\text { UEs/safety V- } \\
\text { UEs) }\end{array}$ & Freeway & $\begin{array}{l}\text { W-UEs } \\
\text { C-UEs } \\
\text { Safety and } \\
\text { nonsafety } \\
\text { V-UEs }\end{array}$ & SINR & - & $\begin{array}{l}\text { Orthogonal RBs } \\
\text { are allocated to } \\
\text { each user type }\end{array}$ & $\begin{array}{l}\text { Kuhn-Munkres } \\
\text { algorithm } \\
\text { Gale-Shapley } \\
\text { algorithm }\end{array}$ & $\begin{array}{c}1 \mathrm{C}-\mathrm{UE} \\
1 \\
\text { nonsafety } \\
\mathrm{V} \text {-UE }\end{array}$ \\
\hline [29] & Unicast & $\begin{array}{l}\text { Maximize the } \\
\text { total } \\
\text { throughput for } \\
\text { C-UEs and V- } \\
\text { UEs }\end{array}$ & Freeway & $\begin{array}{l}\text { W-UEs } \\
\text { C-UEs } \\
\text { Safety and } \\
\text { nonsafety } \\
\text { V-UEs }\end{array}$ & SINR & - & $\begin{array}{l}\text { Orthogonal RBs } \\
\text { are allocated to } \\
\text { each user type }\end{array}$ & $\begin{array}{l}\text { An interior point } \\
\text { method } \\
\text { Kuhn-Munkres } \\
\text { method }\end{array}$ & $\begin{array}{c}1 \mathrm{C}-\mathrm{UE} \\
1 \\
\text { nonsafety } \\
\mathrm{V}-\mathrm{UE}\end{array}$ \\
\hline [30] & Unicast & $\begin{array}{l}\text { Maximize V2I- } \\
\text { UEs sum rate; } \\
\text { guarantee } \\
\text { V2V-UEs } \\
\text { reliability } \\
\text { requirement }\end{array}$ & Freeway & $\begin{array}{l}\text { V2I-UEs } \\
\text { V2V-UEs }\end{array}$ & $\begin{array}{l}\text { SINR } \\
\text { Buffer size } \\
\text { Packet } \\
\text { delay }\end{array}$ & - & $\begin{array}{l}\text { Orthogonal RBs } \\
\text { are allocated to } \\
\text { each user type }\end{array}$ & - & $\begin{array}{l}1 \mathrm{~V} 2 \mathrm{I}-\mathrm{UE} \\
1 \mathrm{~V} 2 \mathrm{~V}-\mathrm{UE}\end{array}$ \\
\hline$[31]$ & Unicast & $\begin{array}{l}\text { Maximize V2I- } \\
\text { UEs sum rate; } \\
\text { guarantee } \\
\text { V2V-UEs } \\
\text { reliability } \\
\text { requirement }\end{array}$ & Freeway & $\begin{array}{l}\text { V2I-UEs } \\
\text { V2V-UEs }\end{array}$ & $\begin{array}{l}\text { SINR } \\
\text { Buffer size } \\
\text { Packet } \\
\text { delay }\end{array}$ & $\mathrm{PC}$ & $\begin{array}{l}\text { Orthogonal RBs } \\
\text { are allocated to } \\
\text { each user type }\end{array}$ & - & $\begin{array}{l}1 \mathrm{~V} 2 \mathrm{I}-\mathrm{UE} \\
1 \mathrm{~V} 2 \mathrm{~V}-\mathrm{UE}\end{array}$ \\
\hline$[32]$ & Unicast & $\begin{array}{l}\text { Maximize C- } \\
\text { UEs sum rate; } \\
\text { guarantee V- } \\
\text { UEs reliability } \\
\text { requirement }\end{array}$ & Freeway & $\begin{array}{l}\text { GBR C- } \\
\text { UEs } \\
\text { NGBR C- } \\
\text { UEs } \\
\text { V-UEs }\end{array}$ & $\begin{array}{l}\text { PDR } \\
\text { SINR } \\
\text { Buffer size } \\
\text { Packet } \\
\text { delay }\end{array}$ & - & $\begin{array}{l}\text { Orthogonal RBs } \\
\text { are allocated to } \\
\text { each user type }\end{array}$ & - & $\begin{array}{l}1 \mathrm{C}-\mathrm{UE} \\
1 \mathrm{~V}-\mathrm{UE}\end{array}$ \\
\hline [33] & Unicast & $\begin{array}{l}\text { Maximize sum } \\
\text { rate and } \\
\text { respect } \\
\text { constraint } \\
\text { delay of C- } \\
\text { UEs; guarantee } \\
\text { V-UEs } \\
\text { reliability and } \\
\text { latency }\end{array}$ & Freeway & $\begin{array}{l}\text { C-UEs } \\
\text { Safety V- } \\
\text { UE } \\
\text { Nonsafety } \\
\text { V-UE }\end{array}$ & $\begin{array}{l}\text { PDOR } \\
\text { SINR } \\
\text { Delay }\end{array}$ & - & $\begin{array}{l}\text { Orthogonal RBs } \\
\text { are allocated to } \\
\text { each user type }\end{array}$ & - & $\begin{array}{c}1 \text { C-UE } \\
1 \text { safety V- } \\
\text { UE } \\
1 \\
\text { nonsafety } \\
\text { V-UE }\end{array}$ \\
\hline
\end{tabular}

taking into account the interference between diverse communication links. The resource-sharing problem has been solved using the graph theory that develops two interference graph-based resource-sharing schemes: the interference-aware and the interference-classified graphbased resource-sharing scheme.
The authors in [19-22] proposed a radio resources and power allocation algorithm for safety-critical vehicular communications. This algorithm aims to maximize the $\mathrm{C}$-UE sum rate with proportional bandwidth fairness under the constraint of satisfying the V-UEs' requirements on latency and reliability. First, they mathematically formulate 
the requirement of $\mathrm{V} 2 \mathrm{X}$ communication into optimization constraints that compute with only slowly varying CSI. Then, they propose a RRM to decide which users can share the same RB.

In [19], a two-step resources allocation algorithm was investigated. Firstly, resources are allocated to both C-UEs and $\mathrm{V}$-UEs in an optimal way by allowing equal power allocation. To communicate with the eNB and among $\mathrm{V}$-UEs, orthogonal RBs are used by C-UEs and V-UEs, respectively. So, both V-UE and C-UE can use the same RB that will produce intracell interference among each other. By transforming the problem of $\mathrm{RB}$ allocation into a maximum weight matching (MWM) problem for bipartite graphs, the interference will be resolved. Secondly, the transmit power is optimally adjusted for each C-UE and V-UE. In [20], the $\mathrm{C}$-UEs sum rate maximized as much as possible and the $\mathrm{V}$-UEs transmit power is minimized.

In [21], a heuristic radio resources allocation scheme was designed considering the fast fading effects in order to support a significantly higher number of V-UEs by allowing nonorthogonality among V-UEs. Resource sharing can take place not only between vehicles and cellular users but also among different vehicles on condition that the SINR constraints of V-UEs and the best rate of C-UEs are satisfied. To do this, they will first introduce the underlaying mode using the Perron-Frobenius theory to design an RB sharing metric. Second, they propose a heuristic RB sharing scheme that associates in a sequential fashion each V-UE with a $\mathrm{C}$-UE. Finally, the power is allocated based on the RB allocation. Then, they extend in [22] to improve more strict latency and reliability requirement designed based on matching theory in order to obtain higher performance especially for high load scenario.

In [23], a radio resource allocation algorithm based on $\mathrm{RBs}$ sharing was proposed in order to maximize the concurrent $\mathrm{V} 2 \mathrm{~V}$ transmissions number unlike other authors who maximize the sum rate, where one $\mathrm{RB}$ can be shared by multiple V-UEs by allowing nonorthogonal access among them. Firstly, the reliability requirement is transformed into constraint of spectral radios matrix to limit the interference among V-UEs. Secondly, they mathematically formulate the $\mathrm{RB}$ sharing problem that maximizing the number of $\mathrm{V}-\mathrm{UEs}$, which is equivalent to minimize the occupied RBs number. To better improve the spectrum efficiency, they use the spectral radius estimation theory.

In [24], Wei et al. proposed a 3D-matching-based radio resources allocation algorithm for $\mathrm{V} 2 \mathrm{X}$ communication. The objective is to maximize the total throughput of nonsafety $\mathrm{V}$-UEs on condition of satisfying the SINR requirements on $\mathrm{C}$-UEs and on safety V-UEs. They proposed three stages to allow these UEs to share the same RB on condition that one $\mathrm{RB}$ cannot be shared by more than one UE in the same type. In the first stage, they obtain the data rate for each nonsafety $\mathrm{V}$-UE in all possible combinations for each RB. In the second stage, they construct the hypergraph model that represents all the combinations of these users to find the set of the largest sum weight of hyperedge obtained from the first stage. Then, in the third stage, the resources allocation matrix was founded based on k-claw.
In [25], the authors proposed a robust radio resource and power allocation scheme for V2X communication in order to maximize the sum throughput of all V2I links while guaranteeing the reliability of each V2V link. A low-complexity algorithm was designed to find the optimal strategy of spectrum sharing among $\mathrm{V} 2 \mathrm{~V}$ and V2I links while adjusting their transmit powers. Firstly, they mathematically formulate the optimized problem to meet the V2V and V2I requirements where one V2I-UE shares spectrum with one V2V-UE. Secondly, a theorem is described in order to obtain the optimal power allocation that maximizes the capacity for V2I-UEs when it shares RB with V2V-UEs, while guaranteeing the minimum capacity requirement of V2I. Then, the Hungarian method is used to find the optimal resources reuses.

In [26], Liang et al. proposed a spectrum sharing resources and power allocation algorithm based only on slowly varying large-scale fading information of wireless channels over the fast fading. The algorithm objective is to maximize the V2I-UE ergodic capacity while guaranteeing the reliability requirement for each V2V-UEs. This algorithm was proposed to support both types of vehicular connections, i.e., V2I and V2V links, where the resource sharing happens between V2V-UEs and V2I-UEs. First, each V2V-UE is paired with each corresponding V2I-UE that satisfies the minimum capacity requirement. Then, the optimal spectrum sharing is found between the V2V-UEs and V2I-UEs sets by constructing a bipartite graph using the Hungarian method.

In [27], Mei et al. investigated a power and resource allocation scheme to jointly optimize the V2V communications. This scheme aims at maximizing the C-UEs information rate and at guaranteeing the reliability and the latency requirements of $\mathrm{V}$-UEs. The latency packets are considered as the most important requirement rather than the data rate. Firstly, they mathematically formulate the endto-end latency packets and transform it into data rate constraint. To guarantee this requirement, a minimum amount of RBs must be assigned to each V-UE. Then, the Lagrange dual decomposition method is applied to find the optimal solution of RBs sharing where at most one $\mathrm{V}$-UE can share the RB that is assigned to $\mathrm{C}-\mathrm{UE}$.

In [28], Wei et al. proposed a joint resource sharing, power control and resource allocation for V2X communication over unlicensed spectrum aiming at guaranteeing fair coexistence among C-UEs, WiFi-UEs, and V-UEs. The latter is classified into nonsafety and safety $\mathrm{V}$-UEs. The safety $\mathrm{V}$-UEs require high reliable services in terms of latency and rate, so they are allowed to use the licensed spectrum. In case of licensed spectrum shortage, they can use the unlicensed spectrum, whereas the nonsafety V-UEs can select to use unlicensed spectrum over Content-Free Period (CFP) LTE$\mathrm{U}$ or CP-based WiFi modes. Therefore, a low complexity scheme is designed in order to maximize the total throughput for nonsafety V-UEs and C-UEs.

In [29], the authors proposed a joint resource sharing and power allocation scheme for heterogeneous vehicular environment over LTE-U. This scheme aims at maximizing the total throughput for C-UEs and V-UEs, where V-UEs are 
categorized into nonsafety and safety V-UEs. The safety V-UEs are allowed to allocate orthogonal RB from the licensed spectrum without sharing with C-UEs in order to guarantee the safety V-UEs reliability. However, the nonsafety $\mathrm{V}$-UEs can allocate RB into two modes. With slow vehicle speed, the nonsafety $\mathrm{V}$-UEs compete $\mathrm{RB}$ with $\mathrm{W}$-UEs from unlicensed spectrum during the $\mathrm{CP}$ interval. With high vehicle speed, the nonsafety V-UEs use the reserved Content-Free Period (CFP) based on LTE-U.

In our previous work [30], radio resource management was investigated for $\mathrm{V}$-UEs where both $\mathrm{V} 2 \mathrm{~V}$ and $\mathrm{V} 2 \mathrm{I}$ communication exist. A resource allocation algorithm is proposed aiming at promising the V2V-UEs reliability requirement and at maximizing the V2I-UEs sum rate. Firstly, V-UEs are separated into two user types; the V2V-UEs and the V2I-UEs, which are sorted in the TD scheduler according to its corresponding metric. Then, in the FD scheduler, RBs are allocated to V2I-UEs by maximizing their sum rate and to V2V-UEs by ensuring their SINR constraint in condition that at most, one V-UEs from each category can share the same resources. In [31], we add a power control mechanism to minimize the interference caused by the $\mathrm{V} 2 \mathrm{~V}$ UEs when sharing RBs with V2I-UEs. Therefore, not only the SINR constraint is considered but also the V2V-UEs power is controlled.

In [32], we designed an efficient scheduling and resources allocation scheme for both V-UEs and C-UEs communications in order to improve C-UEs sum rate and respecting V-UEs latency constraint and C-UEs Packet Drop Rate (PDR) constraint. We classify users into three classes: the V-UEs class, the GBR C-UEs class, and the NGBR C-UEs class. Firstly, all classes' packets are prioritized according to their QoS requirement. Secondly, based on the PDR ratio, resources are dynamically adjusted for GBR and NGBR $\mathrm{C}$-UEs. Then, resources that already allocated to C-UEs are reused by the V-UEs.

In [33], we proposed a mixed resource allocation and traffic sharing among C-UEs, nonsafety V-UEs, and safety V-UEs in order to promise the reliability and latency requirements. The main goal of our proposed is to regard the safety V-UEs and the C-UEs delay constraint and the SINR of all users while maximizing the C-UEs sum rate. After allocating RBs to C-UEs, resources are reused by the nonsafety and safety V-UEs where at most one RB can be shared by three users from different classes taking into consideration each class requirements.

5.1.2. RBs Sharing-Based User Clustering/Grouping. In this section, we investigate the underlaying resource allocation allowing V-UEs to share the same RBs based on user grouping/clustering. The comparison of these algorithms is summarized in Table 2.

In [34], a novel proximity-aware resource allocationbased QoS was designed for V2V-UEs in order to reduce the total power transmission considering the reliability and the queuing latency requirements. The authors exploited the spatial-temporal aspects of V-UEs based on their physical proximity and traffic demands. First of all, clustering mechanism is demonstrated to gather V-UEs into several zones based on their physical proximity. Therefore, dedicated RBs are allocated to each zone based on their traffic demands and QoS requirements. Secondly, within each zone, a power minimization solution based on the leveraging Lyapunov optimization techniques is proposed for each V2V-UE pair.

In [35], the authors proposed a graph-based resource allocation algorithm for broadcast $\mathrm{V} 2 \mathrm{~V}$ communications aiming at maximizing the sum-rate capacity of the system. Many broadcast communication clusters are gathered. Within any cluster, V-UEs should transmit in orthogonal RBs to avoid conflicts but they cannot receive and transmit simultaneously. To prevent conflicts, resources should be allocated in different subframes. However, V-UEs in different clusters can share the same RBs. Therefore, they introduce a bipartite graph-based solution aiming to assign every V-UE with a RB that attains the maximum sum rate.

In [36], the authors proposed a hybrid-scheduling algorithm for geographical zone aiming at maximizing the sum rate of the transmitting V-UEs while taking into account the reliability for all receiving V-UEs. They mathematically model their objective function problem to meet each V-UEs service requirements and to resolve it by adopting the greedy algorithm. Firstly, a hybrid scheduling is applied to allocate resource for V-UEs. The V-UEs in each geographical zone are gathered into groups based on their geographical locations. Secondly, the reuse patterns are defined where resources are reused in each reuse pattern. Then, specific RBs are allocated to V-UEs in each zone, and they will be reused in each reuse pattern.

In [37], Liang et al. studied and presented a graph-based resource allocation for V2V-UEs-based direct link and V2IUEs-based cellular link to improve the sum V2I-UEs and to guarantee theV2V-UEs reliability. Firstly, they mathematically model the resource and the power allocation problem that meets the QoS requirements for V2I-UEs and V2VUEs. Secondly, a graph partitioning algorithm is exploited to address this problem which can be converted into a weighted 3 -dimensional matching problem. The main objective is to gather V2V-UEs into many clusters based on their mutual interferences. Then, all V2V-UEs belonging to the same cluster are allowed to share the same RBs with the corresponding V2I-UEs, whereas V2V-UEs in different clusters are not allowed to share the same RBs.

5.1.3. RBs Sharing-Based User Geographic Location. In this section, we investigate the underlaying resource allocation algorithms that allow V-UEs to share the same RBs based on user geographic location. The comparison of these algorithms is summarized in Table 3.

In [38], Yang et al. proposed a two-stage resource allocation in a dense urban in order to satisfy the data rate and the reliability requirements for both nondelay sensitive services and delay-sensitive services while minimizing the delay-sensitive services latency. The delaysensitive V-UEs utilize LTE-based D2D link, whereas the nondelay-sensitive V-UEs can utilize LTE-C link. The 
TABLE 2: Comparison of the existing underlaying RBs sharing based-user clustering/grouping in V2X services.

\begin{tabular}{|c|c|c|c|c|c|c|c|c|c|}
\hline Ref. & $\begin{array}{l}\text { Unicast/ } \\
\text { broadcast }\end{array}$ & Objectives & Scenarios & $\begin{array}{l}\text { User } \\
\text { types }\end{array}$ & $\begin{array}{l}\text { Allocation } \\
\text { constraints }\end{array}$ & $\begin{array}{c}\text { Power } \\
\text { control/ } \\
\text { allocation }\end{array}$ & Allocation process & Methods/theory & $\begin{array}{c}\mathrm{RB} \\
\text { sharing }\end{array}$ \\
\hline$[34]$ & Unicast & $\begin{array}{l}\text { Minimize the total } \\
\text { power } \\
\text { transmission and } \\
\text { latency reliability }\end{array}$ & Urban & V-UEs & $\begin{array}{l}\text { Queue } \\
\text { length }\end{array}$ & PA & $\begin{array}{l}\text { Nonorthogonal } \\
\text { RBs are allocated } \\
\text { for V2V-UEs in } \\
\text { different clusters }\end{array}$ & $\begin{array}{c}\text { Karush-Kuhn-Tucker } \\
\text { theory }\end{array}$ & $\begin{array}{l}\mathrm{N} \\
\mathrm{V} 2 \mathrm{~V}- \\
\mathrm{UEs}\end{array}$ \\
\hline [35] & Broadcast & $\begin{array}{l}\text { Maximizing the } \\
\text { sum rate capacity } \\
\text { of the system }\end{array}$ & - & V-UEs & Capacity & - & $\begin{array}{l}\text { Nonorthogonal } \\
\text { RBs are allocated to } \\
\text { user in different } \\
\text { clusters }\end{array}$ & $\begin{array}{l}\text { Kuhn-Munkres } \\
\text { method }\end{array}$ & $\begin{array}{l}\text { N V- } \\
\text { UEs }\end{array}$ \\
\hline$[36]$ & Broadcast & $\begin{array}{l}\text { Maximize the sum } \\
\text { rate of the tr. V- } \\
\text { UEs reliability for } \\
\text { rx. V-UEs }\end{array}$ & Highway & $\begin{array}{l}\text { V2V- } \\
\text { UEs }\end{array}$ & OP & - & $\begin{array}{l}\text { Orthogonal RBs } \\
\text { are allocated in } \\
\text { each zone } \\
\text { Nonorthogonal } \\
\text { RBs are allocated in } \\
\text { different zones } \\
\text { Nonorthogonal }\end{array}$ & Greedy algorithm & $\begin{array}{c}\mathrm{N} \\
\mathrm{V} 2 \mathrm{~V}- \\
\mathrm{UEs}\end{array}$ \\
\hline [37] & Unicast & $\begin{array}{c}\text { Maximize the sum } \\
\text { V2I reliability of } \\
\text { V2V }\end{array}$ & $\begin{array}{l}\text { Multilane } \\
\text { freeway }\end{array}$ & $\begin{array}{l}\text { V2V- } \\
\text { UEs } \\
\text { V2I- } \\
\text { UEs }\end{array}$ & OP & PA & $\begin{array}{l}\text { RBs are allocated to } \\
\text { V2V-UEs in the } \\
\text { same cluster } \\
\text { Orthogonal RB is } \\
\text { allocated to V2I- } \\
\text { UEs }\end{array}$ & Graph theory & $\begin{array}{l}1 \text { V2I- } \\
\text { UEs } \\
\mathrm{N} \\
\text { V2V- } \\
\text { UEs }\end{array}$ \\
\hline
\end{tabular}

TABLE 3: Comparison of the existing underlaying resource allocation in V2X services.

\begin{tabular}{|c|c|c|c|c|c|c|c|c|c|}
\hline Ref. & $\begin{array}{c}\text { Unicast/ } \\
\text { broadcast }\end{array}$ & Objectives & Scenarios & User types & $\begin{array}{l}\text { Allocation } \\
\text { constraints }\end{array}$ & $\begin{array}{c}\text { Power } \\
\text { control/ } \\
\text { allocation }\end{array}$ & $\begin{array}{l}\text { Allocation } \\
\text { process }\end{array}$ & Methods/theory & $\begin{array}{c}\mathrm{RB} \\
\text { sharing }\end{array}$ \\
\hline$[38]$ & Broadcast & $\begin{array}{l}\text { Minimize the } \\
\text { delay and } \\
\text { reliabilit } \\
\text { Maximize data } \\
\text { rate }\end{array}$ & $\begin{array}{l}\text { Dense urban } \\
\text { Intersection }\end{array}$ & $\begin{array}{l}\text { V-UEs } \\
\text { (nondelay- } \\
\text { and delay- } \\
\text { sensitive) }\end{array}$ & $\begin{array}{l}\text { Avg. Que. } \\
\text { Leng. PRR }\end{array}$ & - & $\begin{array}{l}\text { Orthogonal RBs } \\
\text { are allocated to } \\
\text { each user type }\end{array}$ & $\begin{array}{c}\text { Traffic flow } \\
\text { theory }\end{array}$ & $\begin{array}{c}1 \\
\text { nondelay- } \\
\text { sensitive } \\
1 \text { delay- } \\
\text { sensitive }\end{array}$ \\
\hline [39] & Unicast & $\begin{array}{c}\text { Minimize } \\
\text { network cost }\end{array}$ & Freewayurban & V-UEs & SINR & - & $\begin{array}{l}\text { Nonorthogonal } \\
\text { RBs are } \\
\text { allocated to V- } \\
\text { UEs }\end{array}$ & $\begin{array}{l}\text { Hare-Niemeyer } \\
\text { method } \\
\text { Matching theory }\end{array}$ & N V-UEs \\
\hline [40] & Unicast & $\begin{array}{l}\text { Reduce the } \\
\text { signaling } \\
\text { overhead and } \\
\text { interference }\end{array}$ & Urban & $\begin{array}{l}\text { C-UEs } \\
\text { V-UEs }\end{array}$ & SINR & - & $\begin{array}{c}\text { Orthogonal RBs } \\
\text { are allocated to } \\
\text { each user type }\end{array}$ & - & $\begin{array}{l}1 \mathrm{C}-\mathrm{UE} \\
1 \mathrm{~V}-\mathrm{UE}\end{array}$ \\
\hline$[41]$ & Unicast & $\begin{array}{l}\text { Reduce the } \\
\text { signaling } \\
\text { overhead and } \\
\text { interference }\end{array}$ & Urban & $\begin{array}{l}\text { C-UEs } \\
\text { V-UEs }\end{array}$ & SINR & $\mathrm{PC}$ & $\begin{array}{l}\text { Orthogonal RBs } \\
\text { are allocated to } \\
\text { each user type }\end{array}$ & Graph theory & $\begin{array}{l}1 \mathrm{C}-\mathrm{UE} \\
1 \mathrm{~V}-\mathrm{UE}\end{array}$ \\
\hline
\end{tabular}

intersection is split into four subregions in order to reduce the complexity. In the first stage, for each subregion, resources are allocated based on the Traffic Density Information (TDI), where orthogonal resources are allocated in different subregions. In the second stage, based on the Channel/Queue State Information (CSI/QSI), reusable resources are used among subregions.

In [39], a dynamic resource allocation in V2V communications is proposed with proximity awareness. This proposed algorithm aims to minimize the total network cost and to maintain successful transmissions while satisfying the QoS requirements for V-UEs. The total network cost is calculated according to traffic load and successful transmission. First, they proposed a dynamic clustering scheme to group the V-UE pairs with similar characteristics into sets of zones based on traffic load and mutual interference using the Hare-Niemeyer method to calculate the set of V-UEs in each zone, where $\mathrm{V}$-UEs can reuse resources in each zone while simultaneously satisfying their QoS. Each zone has a dynamic size and changes over time according to the traffic load and proximity information. Then, a novel intrazone coordination mechanism is described based on a matching game in order to allocate resources among V-UEs in each zone. 
TABLE 4: Comparison of the existing overlaying resource allocation in V2X services.

\begin{tabular}{|c|c|c|c|c|c|c|c|c|}
\hline Ref. & $\begin{array}{c}\text { Unicast/ } \\
\text { broadcast }\end{array}$ & Objectives & Scenarios & User types & $\begin{array}{l}\text { Allocation } \\
\text { constraints }\end{array}$ & Allocation process & Methods/theory & $\begin{array}{c}\mathrm{RB} \\
\text { sharing }\end{array}$ \\
\hline$[42]$ & Broadcast & $\begin{array}{l}\text { Improve resource } \\
\text { utilization } \\
\text { efficiency }\end{array}$ & Highway & V-UEs & $\begin{array}{c}\text { Reuse } \\
\text { distance }\end{array}$ & $\begin{array}{l}\text { Orthogonal resource is } \\
\text { allocated to users whose } \\
\text { relative distance is less } \\
\text { than resource reuse } \\
\text { distance }\end{array}$ & - & N V-UEs \\
\hline$[43]$ & - & - & $\begin{array}{l}\text { Urban/ } \\
\text { freeway }\end{array}$ & $\begin{array}{l}\text { Periodic } \\
\text { traffic of V- } \\
\text { UEs }\end{array}$ & - & $\begin{array}{l}\text { Orthogonal RB are } \\
\text { allocated for each user }\end{array}$ & - & $1 \mathrm{~V}$-UEs \\
\hline [44] & Unicast & $\begin{array}{l}\text { Minimize the total } \\
\text { latency }\end{array}$ & $\begin{array}{l}\text { Multilane } \\
\text { highway }\end{array}$ & V2I-UEs & SINR & $\begin{array}{c}\text { Orthogonal RBs are } \\
\text { allocated to each V-UEs } \\
\text { using D2D } \\
\text { communication over C- } \\
\text { V2X or } 802.11 \mathrm{p}\end{array}$ & Greedy algorithm & $1 \mathrm{~V} 2 \mathrm{I}-\mathrm{UB}$ \\
\hline [45] & Unicast & $\begin{array}{l}\text { Improve network } \\
\text { sum rate }\end{array}$ & Freeway & $\begin{array}{l}\text { C-UEs } \\
\text { V-UEs }\end{array}$ & $\begin{array}{l}\text { Fairness } \\
\text { index } \\
\text { OP }\end{array}$ & $\begin{array}{c}\text { Orthogonal RBs are } \\
\text { allocated to C-UEs and V- } \\
\text { UEs }\end{array}$ & $\begin{array}{l}\text { Ant colony } \\
\text { optimization } \\
\text { mechanism }\end{array}$ & $\begin{array}{l}1 \mathrm{~V}-\mathrm{UE} \\
\text { or } 1 \mathrm{C}- \\
\mathrm{UE}\end{array}$ \\
\hline
\end{tabular}

In [40], Botsov et al. designed a centralized scheduling mechanism based on the position of the vehicles within a single cell. They aim to guarantee continuous transmission for $\mathrm{V} 2 \mathrm{~V}$ link while reducing the interference and the signaling overhead within the primary network and to satisfy the $\mathrm{V} 2 \mathrm{~V}$ safety services requirements. The principle is to divide the cell coverage into several zones, and a set of RBs were assigned to each zone and is dedicated for D2D communication. These sets of RBs are reused by C-UEs while maintaining a minimum distance zone and are chosen according to RB availability and data rate requirements of the V2V service. Then, they extended to cover multicellular deployments in [41]. The zone layout and RB sets are then fixed and do not change over time.

5.2. Overlaying Resource Allocation in V2X Services. Different from the underlaying resource allocation algorithms studied in the previous section, the authors in [42-45] proposed to dedicate specific resources for V2V communications. These proposed resources remove the concerns of interference among $\mathrm{V}$-UEs and C-UEs communications and on the other hand decrease the total achievable resources for cellular communications and accordingly lead in resources starvation. The comparison of the overlaying $\mathrm{V} 2 \mathrm{X}$ resource allocation algorithms is summarized in Table 4.

In [42], Zhang et al. proposed two resource allocation schemes based on V-UEs locations for V2V broadcast services in order to improve RBs utilization and transmission precision in an efficient way and to minimize the time delay. The first scheme is the centralized scheduler, where RBs are allocated to V-UEs in orthogonal way to avoid the cofrequency interference. These RBs are reused in condition that the V-UEs distance is less than resource reuse distance. The distributed scheduler is the second one, where the highway is divided into many areas and RBs are gathered into groups in order to allow V-UEs to select RBs from a specific group in each area.
In [43], Kim et al. proposed a resource allocation scheme based on vehicle direction, position, speed, and density for $\mathrm{V} 2 \mathrm{~V}$ communication. This scheme includes two resource allocation strategies according to vehicle location, the freeway case, and the urban case. A specific resources pool is assigned for each geometric area. For the urban case, high vehicle density occurs in the intersection region, so a special resource was allocated in this region based on traffic density. For the freeway case, resources are allocated based on vehicle direction and position. Each zone of the freeway has a specific resources pool, and when a vehicle enters a zone, it must allocate resources of this zone.

In [44], 802.11p technology and C-V2X communication were investigated in a hybrid resource allocation aiming to improve the reliability of V-UEs and to minimize the total latency. Either using 802.11p or C-V2X interface, a V-UE can transmit packets that will improve the reliability. If the eNB requests a D2D link, V-UEs can transmit using C-V2X interface, if not they will use the $802.11 \mathrm{p}$ interface. In order to improve the latency performance, a set of RBs, periodically, are assigned for V-UEs based D2D link.

In our previous work [45], a swarm intelligence resource allocation algorithm is proposed in order to improve network sum rate while satisfying the QoS requirements for both $\mathrm{V}$-UEs and C-UEs. Firstly, we mathematically express the outage probability as the requirement of the $\mathrm{V}$-UEs and the user fairness index as the requirement of the C-UEs. Secondly, we adaptively (each TTI) assign a set of RBs to the V-UEs and $\mathrm{C}$-UEs where orthogonal RBs are allocated among them. Then, an ant colony optimization (ACO) mechanism is adopted to the resources allocation algorithm to reduce the complexity and to getting a satisfactory performance.

\section{Discussion}

It seems that the V2X resource allocation based-D2D communication algorithms underlaying C-UEs is more popular than the overlaying one. Most of the existing works were proposed in the underlay mode, which are typically designed to avoid the interference problems. However, 
allocating dedicated RBs to V-UEs is not efficient in terms of spectral efficiency compared to the RBs sharing (underlay mode). The latter increases the spectral efficiency whereas RBs might be wasted in the overlay mode. In terms of spectrum sharing, RBs are shared between one C-UE (or V2I-UEs) and at least one V-UE or between more than two $\mathrm{V}$-UEs. Therefore, in the existing resources allocation for V2X based-D2D, nonorthogonal resources are allocated to $\mathrm{V}$-UEs using direct link (V2V-UEs), whereas orthogonal resources are allocated to C-UEs (or V-UEs using direct link (V2I-UEs)).

The focus of the proposed V2X resource allocation algorithms aims to maximize the number of $\mathrm{V} 2 \mathrm{~V}$-UEs that share the same RB with only one C-UE or with only one V2IUE. Then, allocating nonorthogonal resources to C-UEs (or V2I-UEs) can increase the spectral efficiency. Therefore, allocating nonorthogonal access to C-UEs (or V2I-UEs) and nonorthogonal access to $\mathrm{V} 2 \mathrm{~V}$-UEs to share the same RB will be more challenging.

\section{Conclusion}

LTE-D2D is a promising candidate for V2X services which can meet the V2X requirements in terms of latency and reliability. Under LTE-D2D in-band communication, $\mathrm{V}$-UEs can reuse the cellular resources in the underlay mode or in the overlay mode. Hence, it is essential to design a resource allocation algorithm in a way that V-UEs do not affect the C-UEs. V-UEs can communicate using the direct link and share resources with the C-UEs (or V2I-UEs-based cellular link) which may affect the cellular network performance. Consequently, the interference should be managed by resource allocation and power control/allocation algorithms. In this paper, we provide a classification and a comparison of the recent existing resource allocation algorithms for V2X communications.

\section{Conflicts of Interest}

The authors declare that they have no conflicts of interest.

\section{References}

[1] J. Cao, M. Ma, H. Li, Y. Zhang, and Z. Luo, "A survey on security aspects for LTE and LTE-A networks," IEEE Communications Surveys \& Tutorials, vol. 16, no. 1, pp. 283-302, 2014.

[2] K. J. Ahmed and M. J. Lee, "Secure LTE-based V2X service," IEEE Internet of Things Journal, vol. 5, no. 5, pp. 3724-3732, 2018.

[3] 3GPP TR 22.885, v14.0.0, Study on LTE Support for Vehicle to Everything (V2X) Services (Release 14), 3GPP, Valbonne, France, 2015.

[4] 3GPP TR 38.801, V14.0.0, Study on New Radio Access Technology: Radio Access Architecture and Interfaces, 3GPP, Valbonne, France, 2017.

[5] 3GPP TR 38.802, Study on New Radio Access Technology Physical Layer Aspects, 3GPP, Valbonne, France, 2017.

[6] G. Naik, B. Choudhury, and J.-M. Park, "IEEE 802.11 Bd \& 5G NR V2X: evolution of radio access technologies for V2X communications," IEEE Access, vol. 7, pp. 70169-70184, 2019.
[7] A. Asadi, Q. Wang, and V. Mancuso, "A survey on device-todevice communication in cellular networks," IEEE Communications Surveys \& Tutorials, vol. 16, no. 4, pp. 1801-1819, 2014.

[8] P. Mach, Z. Becvar, and T. Vanek, "In-band device-to-device communication in OFDMA cellular networks: a survey and challenges," IEEE Communications Surveys \& Tutorials, vol. 17, no. 4, pp. 1885-1922, 2015.

[9] S. Chen, J. Hu, Y. Shi et al., "Vehicle-to-everything (v2x) services supported by LTE-based systems and 5G," IEEE Communications Standards Magazine, vol. 1, no. 2, pp. 70-76, 2017.

[10] 3GPP TS 22.185, v14.3.0, LTE; Service Requirements for V2X Services, 3GPP, Valbonne, France, 2017.

[11] X. Wang, S. Mao, and M. X. Gong, "An overview of 3GPP cellular vehicle-to-everything standards," GetMobile: Mobile Computing and Communications, vol. 21, no. 3, pp. 19-25, 2017.

[12] 3GPP TS 33.185 V15.0.0, Security Aspect for LTE Support of Vehicle-To-Everything (V2X) Services (Release 15), 3GPP, Valbonne, France, 2018.

[13] R. Molina-Masegosa and J. Gozalvez, "LTE-V for sidelink 5G V2X vehicular communications: a new $5 \mathrm{G}$ technology for short-range vehicle-to-everything communications," IEEE Vehicular Technology Magazine, vol. 12, no. 4, pp. 30-39, 2017.

[14] M. Gonzalez-Martín, M. Sepulcre, R. Molina-Masegosa, and J. Gozalvez, "Analytical models of the performance of C-V2x mode 4 vehicular communications," IEEE Transactions on Vehicular Technology, vol. 68, no. 2, pp. 1155-1166, 2019.

[15] B. Di, L. Song, Y. Li, and G. Y. Li, "Non-orthogonal multiple access for high-reliable and low-latency V2X communications in 5G systems," IEEE Journal on Selected Areas in Communications, vol. 35, no. 10, pp. 2383-2397, 2017.

[16] 3GPP R1-163111, Initial Views and Evaluation Results on Non-orthogonal Multiple Access for NR Uplink, 3GPP, Valbonne, France, 2016.

[17] B. Di, L. Song, Y. Li, and Z. Han, "V2X meets NOMA: nonorthogonal multiple access for 5G-enabled vehicular networks," IEEE Wireless Communications, vol. 24, no. 6, pp. 14-21, 2017.

[18] R. Zhang, X. Cheng, Q. Yao, C.-X. Wang, Y. Yang, and B. Jiao, "Interference graph-based resource-sharing schemes for vehicular networks," IEEE Transactions on Vehicular Technology, vol. 62, no. 8, pp. 4028-4039, 2013.

[19] W. Sun, E. G. Strom, F. Brannstrom, Y. Sui, and K. C. Sou, "D2D-based V2V communications with latency and reliability constraints," in Proceedings of the 2014 IEEE Globecom Workshops (GC Wkshps), pp. 1414-1419, Austin, TX, USA, December 2014.

[20] W. Sun, E. G. Strom, F. Brannstrom, K. C. Sou, and Y. Sui, "Radio resource management for D2D-based V2V communication," IEEE Transactions on Vehicular Technology, vol. 65, no. 8, pp. 6636-6650, 2016.

[21] W. Sun, D. Yuan, E. G. Strom, and F. Brannstrom, "Resource sharing and power allocation for D2D-based safety-critical V2X communications," in Proceedings of the 2015 IEEE International Conference on Communication Workshop (ICCW), pp. 2399-2405, London, UK, June 2015.

[22] W. Sun, D. Yuan, E. G. Strom, and F. Brannstrom, "Clusterbased radio resource management for D2D-supported safetycritical V2X communications," IEEE Transactions on Wireless Communications, vol. 15, no. 4, pp. 2756-2769, 2016.

[23] S. Zhang, Y. Hou, X. Xu, and X. Tao, "Resource allocation in $\mathrm{D} 2 \mathrm{D}$-based $\mathrm{V} 2 \mathrm{~V}$ communication for maximizing the number 
of concurrent transmissions," in Proceedings of the 2016 IEEE 27th Annual International Symposium on Personal, Indoor, and Mobile Radio Communications (PIMRC), pp. 1-6, Valencia, Spain, September 2016.

[24] Q. Wei, W. Sun, B. Bai, L. Wang, E. G. Strom, and M. Song, "Resource allocation for V2X communications: a local search based 3D matching approach," in Proceedings of the 2017 IEEE International Conference on Communications (ICC), pp. 1-6, Paris, France, May 2017.

[25] L. Liang, J. Kim, S. C. Jha, K. Sivanesan, and G. Y. Li, "Spectrum and power allocation for vehicular communications with delayed CSI feedback," IEEE Wireless Communications Letters, vol. 6, no. 4, pp. 458-461, 2017.

[26] L. Liang, G. Y. Li, and W. Xu, "Resource allocation for D2Denabled vehicular communications," IEEE Transactions on Communications, vol. 65, no. 7, pp. 3186-3197, 2017.

[27] J. Mei, K. Zheng, L. Zhao, Y. Teng, and X. Wang, “A latency and reliability guaranteed resource allocation scheme for LTE V2V communication systems," IEEE Transactions on Wireless Communications, vol. 17, no. 6, pp. 3850-3860, 2018.

[28] Q. Wei, L. Wang, Z. Feng, and Z. Ding, "Wireless resource management in LTE-U driven heterogeneous V2X communication networks," IEEE Transactions on Vehicular Technology, vol. 67, no. 8, pp. 7508-7522, 2018.

[29] Q. Wei, L. Wang, Z. Feng, and Z. Ding, "Cooperative coexistence and resource allocation for V2X communications in LTE-unlicensed," in Proceedings of the 2018 15th IEEE Annual Consumer Communications \& Networking Conference (CCNC), pp. 1-6, Las Vegas, NV, USA, January 2018.

[30] A. Masmoudi, S. Feki, K. Mnif, and F. Zarai, "Radio resource allocation algorithm for device to device based on LTE-V2X communications," in Proceedings of the 15th International Joint Conference on e-Business and Telecommunications, pp. 265-271, Porto, Portugal, 2018.

[31] A. Masmoudi, S. Feki, K. Mnif, and F. Zarai, "Efficient radio resource management for D2D- based LTE-V2X communications," in Proceedings of the IEEE/ACS 15th International Conference on Computer Systems and Applications (AICCSA), Aqaba, Jordan, November 2018.

[32] A. Masmoudi, S. Feki, K. Mnif, and F. Zarai, "Efficient scheduling and resource allocation for D2D-based LTE-V2X communications," in Proceedings of the 2019 15th International Wireless Communications \& Mobile Computing Conference (IWCMC), pp. 496-501, Tangier, Morocco, June 2019.

[33] A. Masmoudi, S. Feki, K. Mnif, and F. Zarai, A Mixed Traffic Sharing and Resource Allocation for V2X Communication, CCIS 1118, Chapter 11, 2019.

[34] M. I. Ashraf, C.-F. Liu, M. Bennis, and W. Saad, “Towards low-latency and ultra-reliable vehicle-to-vehicle communication," in Proceedings of the 2017 European Conference on Networks and Communications (EuCNC), pp. 1-5, Oulu, Finland, June 2017.

[35] L. F. Abanto-Leon, A. Koppelaar, and S. H. de Groot, "Graphbased resource allocation with conflict avoidance for V2V broadcast communications," in Proceedings of the 2017 IEEE 28th Annual International Symposium on Personal, Indoor, and Mobile Radio Communications (PIMRC), pp. 1-7, Montreal, Canada, October 2017.

[36] C.-Y. Wei, A. C.-S. Huang, C.-Y. Chen, and J.-Y. Chen, "QoSaware hybrid scheduling for geographical zone-based resource allocation in cellular vehicle-to-vehicle communications," IEEE Communications Letters, vol. 22, no. 3, pp. 610-613, 2018.
[37] L. Liang, S. Xie, G. Y. Li, Z. Ding, and X. Yu, "Graph-based radio resource management for vehicular networks," 2018, http://arxiv.org/abs/1801.02679.

[38] H. Yang, L. Zhao, L. Lei, and K. Zheng, "A two-stage allocation scheme for delay-sensitive services in dense vehicular networks," in Proceedings of the 2017 IEEE International Conference on Communications Workshops (ICC Workshops), pp. 1358-1363, Paris, France, May 2017.

[39] M. I. Ashraf, M. Bennis, C. Perfecto, and W. Saad, "Dynamic proximity-aware resource allocation in vehicle-to-vehicle (V2V) communications," in Proceedings of the 2016 IEEE Globecom Workshops (GC Wkshps), pp. 1-6, Washington, DC, USA, December 2016.

[40] M. Botsov, M. Klugel, W. Kellerer, and P. Fertl, "Location dependent resource allocation for mobile device-to-device communications," in Proceedings of the 2014 IEEE Wireless Communications and Networking Conference (WCNC), pp. 1679-1684, Istanbul, Turkey, April 2014.

[41] M. Botsov, M. Klugel, W. Kellerer, and P. Fertl, "Locationbased resource allocation for mobile D2D communications in multicell deployments," in Proceedings of the 2015 IEEE International Conference on Communication Workshop (ICCW), pp. 2444-2450, London, UK, June 2015.

[42] X. Zhang, Y. Shang, X. Li, and J. Fang, "Research on overlay D2D resource scheduling algorithms for V2V broadcast service," in Proceedings of the 2016 IEEE 84th Vehicular Technology Conference (VTC-Fall), pp. 1-5, Montreal, Canada, September 2016.

[43] J. Kim, J. Lee, S. Moon, and I. Hwang, "A position-based resource allocation scheme for V2V communication," Wireless Personal Communications, vol. 98, no. 1, pp. 15691586, 2018.

[44] F. Abbas and P. Fan, "A hybrid low-latency D2D resource allocation scheme based on cellular V2X networks," in Proceedings of the 2018 IEEE International Conference on Communications Workshops (ICC Workshops), pp. 1-6, Kansas City, MO, USA, May 2018.

[45] S. Feki, A. Masmoudi, A. Belghith, F. Zarai, and M. S. Obaidat, "Swarm intelligence-based radio resource management for D2D-based V2V communication," International Journal of Communication Systems, p. e3817, 2018. 


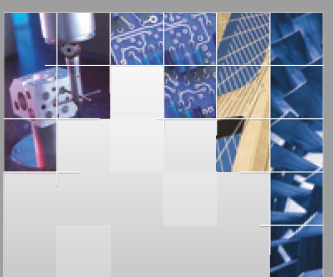

\section{Enfincering}
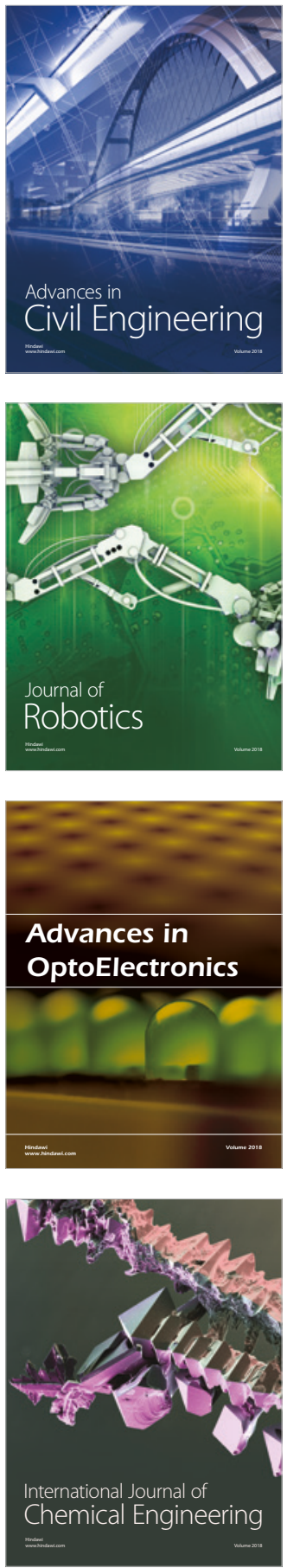

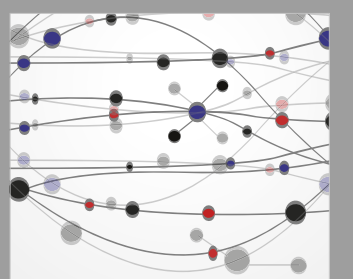

\section{Rotating \\ Machinery}

The Scientific World Journal

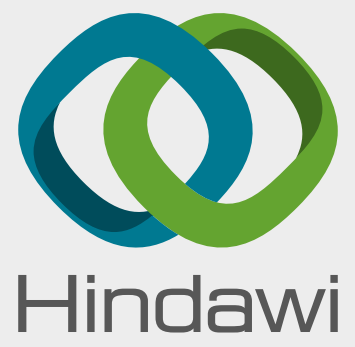

Submit your manuscripts at

www.hindawi.com
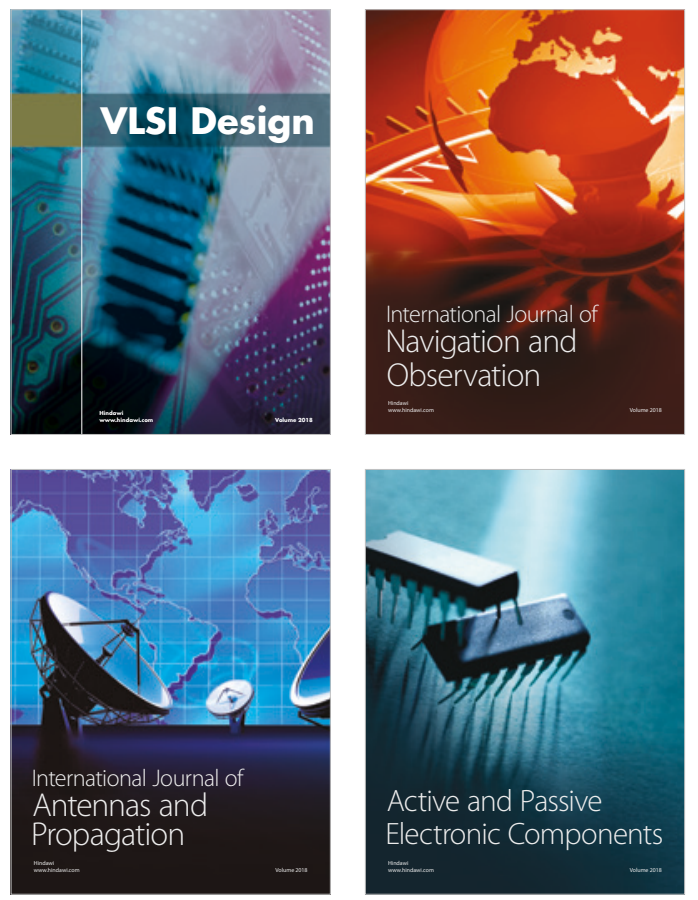
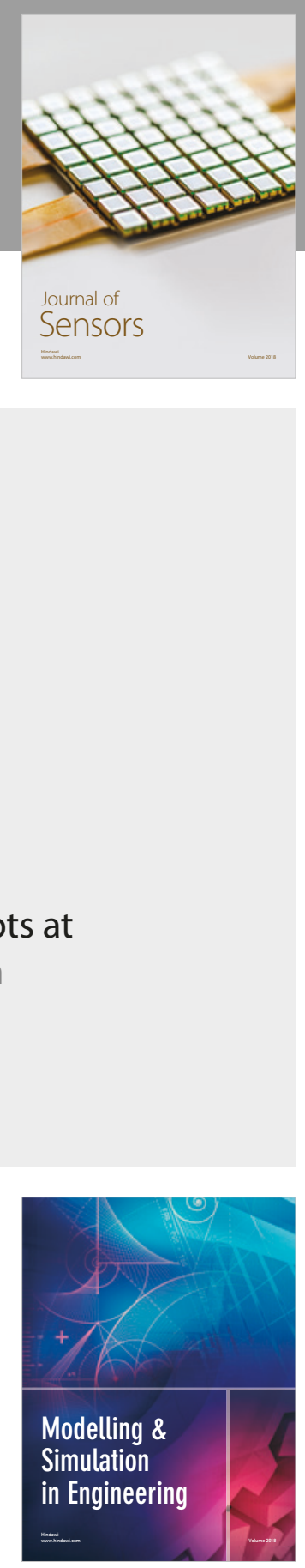

\section{Advances \\ Multimedia}
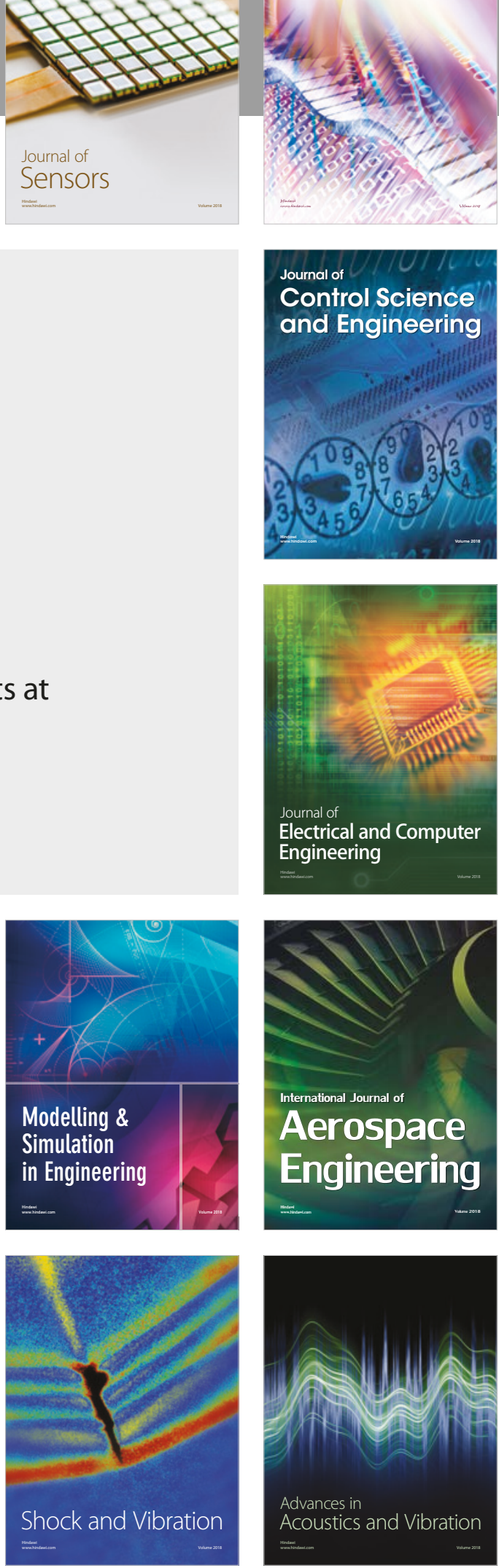